\title{
Thermal transport in the Falicov-Kimball model
}

\author{
J. K. Freericks ${ }^{1}$ and V. Zlatić ${ }^{2}$ \\ ${ }^{1}$ Department of Physics, Georgetown University, Washington, DC 2005\%-0995, U.S.A. \\ ${ }^{2}$ Institute of Physics, Zagreb, Croatia
}

(November 14, 2018)

\begin{abstract}
We prove the Jonson-Mahan theorem for the thermopower of the Falicov-Kimball model by solving explicitly for the correlation functions in the large dimensional limit. We prove a similar result for the thermal conductivity. We separate the results for thermal transport into the pieces of the heat current that arise from the kinetic energy and those that arise from the potential energy. Our method of proof is specific to the Falicov-Kimball model, but illustrates the near cancellations between the kinetic-energy and potential-energy pieces of the heat current implied by the Jonson-Mahan theorem.
\end{abstract}

Primary: 72.15.Jf; 72.20.Pa, 71.20.+h, 71.10.Fd

\section{INTRODUCTION}

The Jonson-Mahanl 1 国 theorem shows that there is a simple relation between the transport coefficient for the electrical conductivity and that needed for the thermopower. The relation is that the integral for the $L_{12}$ coefficient has an extra power of frequency in the integrand than the $L_{11}$ fpefficient. This result has been known for many years 3 for a noninteracting systemthe Jonson-Mahan theorem generalizes this result for a wide class of many-body systems (including the FalicovKimball model, the static Holstein model, and the periodic Anderson model, but not including the Hubbard model or quantum Holstein model).

We use the exact solution of the Falicov-Kimball model in the large-dimensional limit to provide an alternate derivation of the Jonson-Mahan theorem by explicitly evaluating all relevant correlation functions needed for the thermal transport. Our exact analysis also allows us to separate the contributions to thermal transport that arise from the kinetic energy and the potential energy pieces of the heat current. These results provide an interesting interpretation of thermal transport in correlated systems.

In Section II we develop the formalism for deriving the dc conductivity, the thermopower and the thermal conductivity. We derive exact results for the relevant correlation functions and use them to prove the Jonson-Mahan theorem and its generalization to the thermal conductivity. In Section III we provide numerical results for the thermal transport illustrating the different contributions to the thermal coefficients for a number of illustrative cases. Conclusions are presented in Section IV.

\section{FORMALISM FOR THE THERMAL TRANSPORT}

The Hamiltonian for our system is the spin-one-half Falicov-Kimball model

$$
H=-\frac{t^{*}}{2 \sqrt{d}} \sum_{\langle i, j\rangle \sigma} c_{i \sigma}^{\dagger} c_{j \sigma}+E_{f} \sum_{i} w_{i}+U \sum_{i \sigma} w_{i} c_{i \sigma}^{\dagger} c_{i \sigma}
$$

where $c_{i \sigma}^{\dagger}\left(c_{i \sigma}\right)$ is the electron creation (annihilation) operator for an electron at site $i$ with spin $\sigma, E_{f}$ is the energy level of the localized electrons, $w_{i}$ is a variable that equals zero or one and corresponds to the localized electron number, and $U$ is the interaction strength. The hopping integral is scaled with the spatial dimension $d$ so as to have a finite result in the limit $d \rightarrow \infty$; we measure all energies in units of $t^{*}=1$. We work on a hypercubic lattice where the noninteracting density of states is a Gaussian $\rho(\epsilon)=\exp \left(-\epsilon^{2}\right) / \sqrt{\pi}$.

The Falicov-Kimball model can be solved exactly by employing dynamical mean field theoryle.t. Because the self energy $\Sigma(z)$ is local, the local Green's function satisfies

$$
G(z)=\int d \epsilon \rho(\epsilon) \frac{1}{z+\mu-\Sigma(z)-\epsilon},
$$

with $z$ anywhere in the complex plane (we suppress the spin index here). The self energy, local Green's function, and effective medium $G_{0}$ are related by

$$
G_{0}^{-1}(z)-G^{-1}(z)=\Sigma(z),
$$

and the Green's function also satisfies

$$
G(z)=\left(1-w_{1}\right) G_{0}(z)+w_{1} \frac{1}{G_{0}^{-1}(z)-U} .
$$

Here $w_{1}$ is the average concentration of localized electrons,

$$
w_{1}=2 \exp \left[-\beta\left(E_{f}-\mu\right)\right] Z_{\uparrow}(\mu-U) Z_{\downarrow}(\mu-U) / Z,
$$

with $Z=Z_{\uparrow}(\mu) Z_{\downarrow}(\mu)+2 \exp \left[-\beta\left(E_{f}-\mu\right)\right] Z_{\uparrow}(\mu-$ $U) Z_{\downarrow}(\mu-U)$ and

$$
Z_{\sigma}(\mu)=2 e^{\beta \mu / 2} \prod_{n} \frac{i \omega_{n}+\mu-\lambda_{\sigma}\left(i \omega_{n}\right)}{i \omega_{n}} .
$$


The factor of two arises from the spin degeneracy of the f-electrons and the constraint that no more than one felectron is allowed on any site. The symbol $\lambda_{\sigma}(z)$ is defined from the effective medium via $\lambda_{\sigma}\left(i \omega_{n}\right)=i \omega_{n}+\mu-$ $G_{0 \sigma}^{-1}\left(i \omega_{n}\right), \omega_{n}=\pi T(2 n+1)$ is the Fermionic Matsubara frequency, and $\beta=1 / T$. The algorithm for determining the Green's function begins with the self energy set equal to zero. Then Eq. (2) is used to find the local Green's function. The effective medium is found from Eq. (3) and the localized electron filling from Eq. (5). The new local Green's function is then found from Eq. (A) and the new self energy from Eq. (3). This algorithm is repeated until it converges.

Transport properties are calculated within a KuboGreenwood formalism 8 . This relates the transport coefficients to correlation functions of the corresponding transport current operators. We will deal with two current operators here - the particle current 9

$$
\mathbf{j}=\sum_{q \sigma} \mathbf{v}_{q} c_{q \sigma}^{\dagger} c_{q \sigma},
$$

(where the velocity operator is $\mathbf{v}_{q}=\nabla_{q} \epsilon(q)$ and the Fourier transform of the creation operator is $c_{q}^{\dagger}=$ $\left.\sum_{j} \exp \left[i q \cdot \mathbf{R}_{j}\right] c_{j}^{\dagger} / \mathrm{N}\right)$ and the heat current

$$
\begin{aligned}
\mathbf{j}_{Q} & =\sum_{q \sigma}\left(\epsilon_{q}-\mu\right) \mathbf{v}_{q} c_{q \sigma}^{\dagger} c_{q \sigma} \\
& +\frac{U}{2} \sum_{q q^{\prime} \sigma} W\left(q-q^{\prime}\right)\left[\mathbf{v}_{q}+\mathbf{v}_{q^{\prime}}\right] c_{q \sigma}^{\dagger} c_{q^{\prime} \sigma}
\end{aligned}
$$

[where $\left.W(q)=\sum_{j} \exp \left(-i q \cdot \mathbf{R}_{j}\right) w_{j} / N\right]$. The heat current can be broken into two pieces: (i) a kinetic-energy piece $\mathbf{j}_{Q}^{K}$ which is the first term in Eq. (8) and (ii) a potential energy piece $\mathbf{j}_{Q}^{P}$ which is the second term in Eq. (8).

The particle current is defined by the commutator of the Hamiltonian with the polarization operator 9 When evaluated on a lattice with nearest-neighbor hopping, one finds factors that involve the weighted summation of the nearest-neighbor translation vectors $\delta$ weighted by phase factors $\exp (i q \cdot \delta)$, which yield the velocity operator terms above. The definition of the heat-current operator is more involved, and requires the Hamiltonian to be separated into operators $h_{i}$ that involve the site $i$ (in decomposing the kinetic-energy operator into "localized" pieces, one symmetrically assigns half of the $c_{i \sigma}^{\dagger} c_{j \sigma}+c_{j \sigma}^{\dagger} c_{i \sigma}$ term to site $i$ and half to site $\left.j\right)$. These operators can be combined with the position operator to construct an "energy" polarization operator $\sum_{i} \mathbf{R}_{i} h_{i}$, which is commuted with the Hamiltonian to determine the energy current operator; the heat current operator is just this energy current operator shifted by the chemical potential multiplied by the number current operator. Important operator relations between the heat-current operator and the particle-current operator are described fully below [see the discussion around Eq. (60)].
The dc conductivity $\sigma$, thermopower $S$ and electronic thermal conductivity $\kappa$ can all be determined from relevant correlation functions of the current operators. We define three transport coefficients $L_{11}, L_{12}=L_{21}$, and $L_{22}$. Then

$$
\begin{gathered}
\sigma=\frac{e^{2}}{T} L_{11}, \\
S=-\frac{k_{B}}{|e| T} \frac{L_{12}}{L_{11}},
\end{gathered}
$$

and

$$
\kappa=k_{B}^{2}\left[L_{22}-\frac{L_{12} L_{21}}{L_{11}}\right] .
$$

The transport coefficients are found from the analytic continuation of the relevant "polarization operators" at zero frequency

$$
\begin{aligned}
L_{11} & =\lim _{\nu \rightarrow 0} \operatorname{Re} \frac{i}{\nu} \bar{L}_{11}(\nu) \\
\bar{L}_{11}\left(i \nu_{l}\right) & =\pi T \int_{0}^{\beta} d \tau e^{i \nu_{l} \tau}\left\langle T_{\tau} j_{\alpha}^{\dagger}(\tau) j_{\beta}(0)\right\rangle
\end{aligned}
$$

where $\nu_{l}=2 \pi T l$ is the Bosonic Matsubara frequency, the $\tau$-dependence of the operator is with respect to the full Hamiltonian in Eq. (11), and we must analytically continue $\bar{L}_{11}\left(i \nu_{l}\right)$ to the real axis $\bar{L}_{11}(\nu)$ before taking the limit $\nu \rightarrow 0$. Similar definitions hold for the other transport coefficients:

$$
\begin{aligned}
L_{12} & =\lim _{\nu \rightarrow 0} \operatorname{Re} \frac{i}{\nu} \bar{L}_{12}(\nu) \\
\bar{L}_{12}\left(i \nu_{l}\right) & =\pi T \int_{0}^{\beta} d \tau e^{i \nu_{l} \tau}\left\langle T_{\tau} j_{\alpha}^{\dagger}(\tau) j_{Q \beta}(0)\right\rangle
\end{aligned}
$$

and

$$
\begin{aligned}
L_{22} & =\lim _{\nu \rightarrow 0} \operatorname{Re} \frac{i}{\nu} \bar{L}_{22}(\nu) \\
\bar{L}_{22}\left(i \nu_{l}\right) & =\pi T \int_{0}^{\beta} d \tau e^{i \nu_{l} \tau}\left\langle T_{\tau} j_{Q \alpha}^{\dagger}(\tau) j_{Q \beta}(0)\right\rangle .
\end{aligned}
$$

In all of these equations, the subscripts $\alpha$ and $\beta$ denote the respective spatial index of the current vectors.

We begin with a derivation that shows the analytic continuation for the conductivity. Substituting the definition of the particle current operator of Eq. (7) into Eq. (12) for $\bar{L}_{11}$ yields

$$
\begin{aligned}
\bar{L}_{11}\left(i \nu_{l}\right) & =\pi T \int_{0}^{\beta} d \tau e^{i \nu_{l} \tau} \sum_{q q^{\prime} \sigma \sigma^{\prime}} \mathbf{v}_{q \alpha} \mathbf{v}_{q^{\prime} \beta} \\
& \times\left\langle T_{\tau} c_{q \sigma}^{\dagger}(\tau) c_{q \sigma}(\tau) c_{q^{\prime} \sigma^{\prime}}^{\dagger}(0) c_{q^{\prime} \sigma^{\prime}}(0)\right\rangle .
\end{aligned}
$$

The correlation function can be determined from Dyson's equation which relates the dressed correlation function to the bare correlation function via the irreducible charge 
vertex. Since the charge vertex is local in the infinitedimensional limit, it is an even function of momentum, and any sum over momentum that is weighted by just one factor of $\mathbf{v}_{q}$ will vanish. Hence, the dressed correlation fynction is equal to just the bare correlation function 10 (note that the contractions of the operators at equal times also vanish when summed over momentum). This produces

$$
\bar{L}_{11}\left(i \nu_{l}\right)=-\pi T \int_{0}^{\beta} d \tau e^{i \nu_{l} \tau} \sum_{q \sigma} \mathbf{v}_{q \alpha} \mathbf{v}_{q \beta} G_{q \sigma}(\tau) G_{q \sigma}(-\tau)
$$

Now, we introduce the Fourier transform of the Green's function $G(\tau)=T \sum_{n} \exp \left(-i \omega_{n} \tau\right) G_{n}$ with $G_{n}=$ $\int_{0}^{\beta} d \tau \exp \left(i \omega_{n} \tau\right) G(\tau)$. Substituting into Eq. (16), allows us to perform the integral over $\tau$. This finally produces

$$
\bar{L}_{11}\left(i \nu_{l}\right)=-\pi T^{2} \sum_{n} \sum_{q \sigma} \mathbf{v}_{q \alpha} \mathbf{v}_{q \beta} G_{q \sigma}\left(i \omega_{n}\right) G_{q \sigma}\left(i \omega_{n+l}\right) .
$$

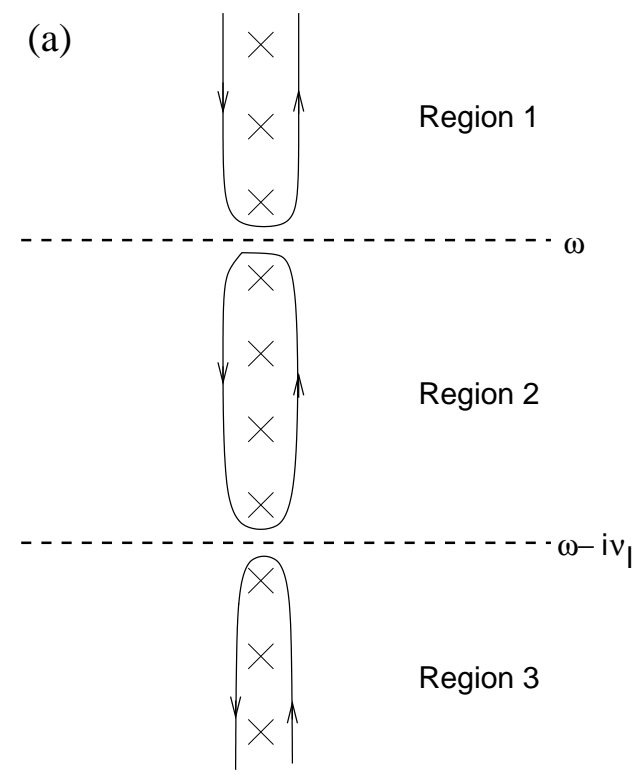

Freericks and Zlatic, Phys Rev B, Figure 1(a)
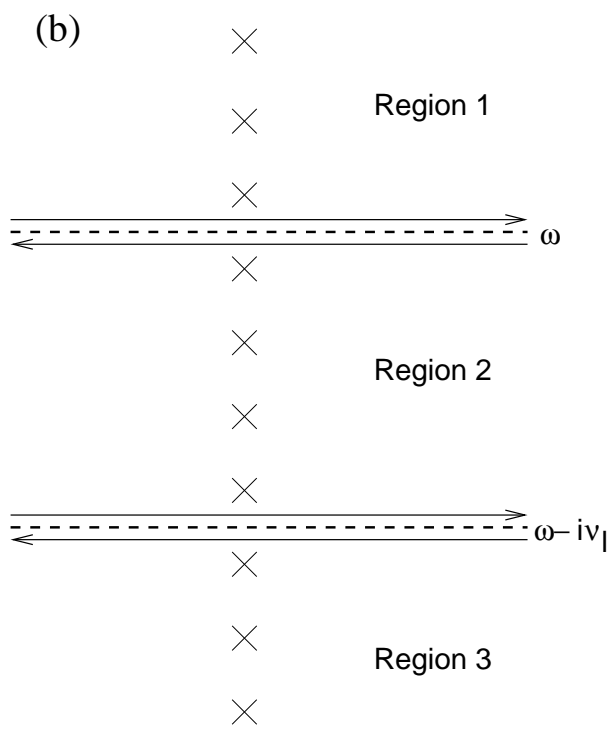

Freericks and Zlatic, Phys. Rev. B, Figure 1(b)

FIG. 1. Contours used in the analytic continuation: (a) contour needed for the Matsubara frequency summation and (b) deformed contour to lines parallel to the real axis.

The next step is to perform the analytic continuation from the imaginary to real axis. The procedure is standard8. We first write the summation over Matsubara frequencies as an integral over the contour $C$ shown in Fig. 1(a) which has contributions at the poles of the Fermi function $f(\omega)=1 /[1+\exp (\beta \omega)]$ which lie at the Fermionic Matsubara frequencies. The contours are then deformed to lines parallel to the real axis, with the Green's functions evaluated with either retarded (R) or advanced (A) functions. The result is

$$
\begin{aligned}
\bar{L}_{11}\left(i \nu_{l}\right)= & -\frac{T}{2 i} \int_{C} d \omega f(\omega) \sum_{q \sigma} \mathbf{v}_{q \alpha} \mathbf{v}_{q \beta} \\
& \times G_{q \sigma}(\omega) G_{q \sigma}\left(\omega+i \nu_{l}\right), \\
= & -\frac{T}{2 i} \int_{-\infty}^{\infty} d \omega f(\omega) \sum_{q \sigma} \mathbf{v}_{q \alpha} \mathbf{v}_{q \beta} \\
& \times\left[G_{q \sigma}^{R}(\omega)-G_{q \sigma}^{A}(\omega)\right] G_{q \sigma}^{R}\left(\omega+i \nu_{l}\right) \\
- & \frac{T}{2 i} \int_{-\infty}^{\infty} d \omega f\left(\omega-i \nu_{l}\right) \sum_{q \sigma} \mathbf{v}_{q \alpha} \mathbf{v}_{q \beta} \\
& \times G_{q \sigma}^{A}\left(\omega-i \nu_{l}\right)\left[G_{q \sigma}^{R}(\omega)-G_{q \sigma}^{A}(\omega)\right] .
\end{aligned}
$$

The analytic continuation is performed by first rewriting $f\left(\omega-i \nu_{l}\right)=f(\omega)$, then taking $i \nu_{l} \rightarrow \nu+i \delta$ and shifting the integration variable $\omega \rightarrow \omega+\nu$ in the second integral. Then, using the definition for $L_{11}$, we finally arrive at

$$
\begin{aligned}
L_{11} & =\lim _{\nu \rightarrow 0}-\frac{T}{2 \nu} \int_{-\infty}^{\infty} d \omega \sum_{q \sigma} \mathbf{v}_{q \alpha} \mathbf{v}_{q \beta} \\
& \times \operatorname{Re}\left\{f(\omega) G_{q \sigma}(\omega) G_{q \sigma}(\omega+\nu)\right. \\
& -f(\omega+\nu) G_{q \sigma}^{*}(\omega) G_{q \sigma}^{*}(\omega+\nu)
\end{aligned}
$$




$$
\left.-[f(\omega)-f(\omega+\nu)] G_{q \sigma}^{*}(\omega) G_{q \sigma}(\omega+\nu)\right\} .
$$

Since $G_{q \sigma}(\omega)=1 /[\omega+\mu-\Sigma(\omega)-\epsilon(q)]$, we can perform the summation over $q$ directly. Because $\epsilon(q)$ is an even function of $q$ and $\mathbf{v}_{q}$ is odd, we must have $\alpha=\beta$. Converting the fraction into the integral of an exponential, then allows the summation over $q$ to be performed directly 11 . The summation over $q$ can be written as an integral over energy with a weighting factor of $\rho(\epsilon) t^{* 2} / d$. This yields

$$
\begin{aligned}
L_{11} & =\lim _{\nu \rightarrow 0}-\frac{T t^{* 2}}{2 \nu d} \delta_{\alpha \beta} \int_{-\infty}^{\infty} d \omega \sum_{\sigma} \int_{-\infty}^{\infty} d \epsilon \rho(\epsilon) \\
& \times \operatorname{Re}\left\{f(\omega) G_{q \sigma}(\omega) G_{q \sigma}(\omega+\nu)\right. \\
& -f(\omega+\nu) G_{q \sigma}^{*}(\omega) G_{q \sigma}^{*}(\omega+\nu) \\
& \left.-[f(\omega)-f(\omega+\nu)] G_{q \sigma}^{*}(\omega) G_{q \sigma}(\omega+\nu)\right\} .
\end{aligned}
$$

If we define $\sigma_{0}=e^{2} t^{* 2} /(2 d)$, and we perform the integral over $\epsilon$, we arrive at

$$
\begin{aligned}
L_{11} & =\lim _{\nu \rightarrow 0} \frac{T \sigma_{0}}{e^{2}} \delta_{\alpha \beta} \int_{-\infty}^{\infty} d \omega \\
& \times \frac{f(\omega)-f(\omega+\nu)}{\nu} \operatorname{Re}\left[-\frac{G(\omega)-G(\omega+\nu)}{\nu+\Sigma(\omega)-\Sigma(\omega+\nu)}\right. \\
& \left.+\frac{G^{*}(\omega)-G(\omega+\nu)}{\nu+\Sigma^{*}(\omega)-\Sigma(\omega+\nu)}\right]
\end{aligned}
$$

The final step is to take the limit of $\nu \rightarrow 0$. Using the facts that

$$
\lim _{\nu \rightarrow 0} \frac{f(\omega)-f(\omega+\nu)}{\nu}=-\frac{d f(\omega)}{d \omega},
$$

and

$$
\lim _{\nu \rightarrow 0} \frac{G(\omega)-G(\omega+\nu)}{\nu+\Sigma(\omega)-\Sigma(\omega+\nu)}=-2+2[\omega+\mu-\Sigma(\omega)] G(\omega),
$$

produces our final result for $L_{11}$

$$
L_{11}=\frac{T \sigma_{0}}{e^{2}} \int_{-\infty}^{\infty} d \omega\left(-\frac{d f(\omega)}{d \omega}\right) \tau(\omega)
$$

with the relaxation time $\tau(\omega)$ defined by

$$
\tau(\omega)=\frac{\operatorname{Im} G(\omega)}{\operatorname{Im} \Sigma(\omega)}+2-2 \operatorname{Re}\{[\omega+\mu-\Sigma(\omega)] G(\omega)\} .
$$

This result appears different from that originally derived for the hypercubic lattice 12 , but Eqs. (24) and (25) do yield the same result; the form presented here has the integral over the noninteracting density of states performed exactly.

The derivation for the transport coefficient $L_{12}$, needed for the thermopower, proceeds in a similar fashion. We can divide the heat current into two pieces, one corresponding to the kinetic energy and one corresponding to the potential energy. This allows us to write $L_{12}=L_{12}^{K}+L_{12}^{P}$. The derivation for the kinetic energy piece follows exactly like the derivation for $L_{11}$ except there is an extra factor of $\epsilon-\mu$ that appears in Eq. (20). The integral over $\epsilon$ can then be performed straightforwardly producing

$$
\begin{aligned}
& L_{12}^{K}=\lim _{\nu \rightarrow 0} \frac{T \sigma_{0}}{e^{2}} \delta_{\alpha \beta} \int_{-\infty}^{\infty} d \omega \frac{f(\omega)-f(\omega+\nu)}{\nu} \\
\times & \operatorname{Re}\left\{-\frac{[\omega-\Sigma(\omega)] G(\omega)-[\omega+\nu-\Sigma(\omega+\nu)] G(\omega+\nu)}{\nu+\Sigma(\omega)-\Sigma(\omega+\nu)}\right. \\
+ & \left.\frac{\left[\omega-\Sigma^{*}(\omega)\right] G^{*}(\omega)-[\omega+\nu-\Sigma(\omega+\nu)] G(\omega+\nu)}{\nu+\Sigma^{*}(\omega)-\Sigma(\omega+\nu)}\right\} .
\end{aligned}
$$

Evaluating the limit $\nu \rightarrow 0$ is simple. The final result is

$$
\begin{aligned}
L_{12}^{K} & =\frac{T \sigma_{0}}{e^{2}} \int_{-\infty}^{\infty} d \omega\left(-\frac{d f(\omega)}{d \omega}\right)\{[\omega-\operatorname{Re} \Sigma(\omega)] \tau(\omega) \\
& -2 \operatorname{Im} \Sigma(\omega) \operatorname{Im}[(\omega+\mu-\Sigma(\omega)) G(\omega)]\}
\end{aligned}
$$

with $\tau(\omega)$ defined in Eq. (25).

The derivation of the potential energy piece is much more involved. The first step is to replace the momentum-dependent operator $W\left(q-q^{\prime}\right)$ by its Fourier transform. Simplifying the expression for $\bar{L}_{12}^{P}$ yields

$$
\begin{aligned}
\bar{L}_{12}^{P}\left(i \nu_{l}\right) & =\frac{\pi T U}{2} \int_{0}^{\beta} d \tau e^{i \nu_{l} \tau} \sum_{q q^{\prime} \sigma \sigma^{\prime}} \frac{1}{N} \sum_{j} \mathbf{v}_{q \alpha} \mathbf{v}_{q^{\prime} \beta} \\
& \times\left[e^{-i q^{\prime} \cdot \mathbf{R}_{j}}\left\langle T_{\tau} w_{j} c_{q \sigma}^{\dagger}(\tau) c_{q \sigma}(\tau) c_{q^{\prime} \sigma^{\prime}}^{\dagger}(0) c_{j \sigma^{\prime}}(0)\right\rangle\right. \\
& \left.+e^{i q^{\prime} \cdot \mathbf{R}_{j}}\left\langle T_{\tau} w_{j} c_{q \sigma}^{\dagger}(\tau) c_{q \sigma}(\tau) c_{j \sigma^{\prime}}^{\dagger}(0) c_{q^{\prime} \sigma^{\prime}}(0)\right\rangle\right]
\end{aligned}
$$

Noting that the $w_{j}$ operator commutes with the Fermionic operators, allows us to use Wick's theorem to rewrite the terms in the square bracket as

$$
\begin{aligned}
\frac{1}{N} \sum_{j} & {\left[-e^{-i q \cdot \mathbf{R}_{j}}\left\langle T_{\tau} w_{j} c_{q \sigma}^{\dagger}(\tau) c_{j \sigma}(0)\right\rangle G_{q \sigma}(\tau)\right.} \\
& \left.+e^{i q \cdot \mathbf{R}_{j}}\left\langle T_{\tau} w_{j} c_{q \sigma}(\tau) c_{j \sigma}^{\dagger}(0)\right\rangle G_{q \sigma}(-\tau)\right] \delta_{q q^{\prime}} \delta_{\sigma \sigma^{\prime}},
\end{aligned}
$$

where we have taken the appropriate contractions (note the velocity operators guarantee that we need not worry about any vertex corrections). The correlation functions in Eq. (29) can be evaluated by taking the derivative with respect to the components of an infinitesimal field $-\sum_{j} h_{j} w_{j}$. These correlation functions have a factor of $\exp [-\beta H]$ in the numerator and a factor of $Z$ in the denominator. In addition, the $\tau$-dependence of the operators arises from factors of $\exp [ \pm \tau H]$. Since the operator $w_{j}$ commutes with all Fermionic operators, it is easy to verify that the expression in Eq. (29) becomes

$$
-\frac{1}{N} \sum_{j}\left[G_{q \sigma}(\tau)\left(T \frac{\partial}{\partial h_{j}}+\left\langle w_{j}\right\rangle\right) G_{q \sigma}(-\tau)\right.
$$




$$
\left.+G_{q \sigma}(-\tau)\left(T \frac{\partial}{\partial h_{j}}+\left\langle w_{j}\right\rangle\right) G_{q \sigma}(\tau)\right] \delta_{q q^{\prime}} \delta_{\sigma \sigma^{\prime}}
$$

which follows by first removing the $w_{j}$ operator through the derivative, then expressing the Fermionic operator at site $j$ through a Fourier transform, and finally evaluating the Fermionic averages. Substituting this result into Eq. (28) then yields

$$
\begin{aligned}
\bar{L}_{12}^{P}\left(i \nu_{l}\right) & =-\frac{\pi T^{2} U}{2 N} \sum_{n} \sum_{q \sigma} \sum_{j} \mathbf{v}_{q \alpha} \mathbf{v}_{q \beta} \\
& \times\left(\left\{\left[T \frac{\partial}{\partial h_{j}}+\left\langle w_{j}\right\rangle\right] G_{q \sigma}\left(i \omega_{n}\right)\right\} G_{q \sigma}\left(i \omega_{n+l}\right)\right. \\
& \left.+G_{q \sigma}\left(i \omega_{n}\right)\left[T \frac{\partial}{\partial h_{j}}+\left\langle w_{j}\right\rangle\right] G_{q \sigma}\left(i \omega_{n+l}\right)\right) .
\end{aligned}
$$

The derivatives need to be computed. Writing the momentum-dependent Green's function as a Fourier transform

$$
G_{q \sigma}\left(i \omega_{n}\right)=\frac{1}{N} \sum_{i-j} e^{i q \cdot\left(\mathbf{R}_{i}-\mathbf{R}_{j}\right)} G_{i j \sigma}\left(i \omega_{n}\right)
$$

and using the identity

$$
G_{i j \sigma}\left(i \omega_{n}\right)=\sum_{k l} G_{i k \sigma}\left(i \omega_{n}\right) G_{k l \sigma}^{-1}\left(i \omega_{n}\right) G_{l j \sigma}\left(i \omega_{n}\right),
$$

(with $G^{-1}$ the matrix inverse of $G$ ) allows us to compute the derivative as

$$
\begin{aligned}
\frac{\partial}{\partial h_{j}} G_{q \sigma}\left(i \omega_{n}\right) & =\frac{1}{N} \sum_{i-j} e^{i q \cdot\left(\mathbf{R}_{i}-\mathbf{R}_{j}\right)} G_{i j \sigma}\left(i \omega_{n}\right) \\
& \times \frac{\partial \Sigma_{j j \sigma}\left(i \omega_{n}\right)}{\partial h_{j}} G_{j j \sigma}\left(i \omega_{n}\right)
\end{aligned}
$$

But in a homogeneous phase, the derivative of the local self energy with respect to the local field, and the local Green's function are both independent of the site $j$, so we finally arrive at

$$
\frac{\partial G_{q \sigma}\left(i \omega_{n}\right)}{\partial h}=G_{n \sigma} \frac{\partial \Sigma_{n \sigma}}{\partial h} G_{q \sigma}\left(i \omega_{n}\right) .
$$

Since the self energy depends only on $G_{n}$ and $w_{1}$, the derivative can be computed by taking partial derivatives and using the chain rule

$$
\frac{\partial \Sigma_{n \sigma}}{\partial h}=\frac{\frac{\partial \Sigma_{n \sigma}}{\partial w_{1}} \frac{\partial w_{1}}{\partial h}}{1-G_{n \sigma}^{2} \frac{\partial \Sigma_{n \sigma}}{\partial G_{n \sigma}}}
$$

Each of the derivatives in Eq. (36) can be found directly 6 .

$$
\begin{gathered}
\frac{\partial \Sigma_{n \sigma}}{\partial w_{1}}=\frac{U}{1+G_{n \sigma}\left(2 \Sigma_{n \sigma}-U\right)}, \\
\frac{\partial w_{1}}{\partial h}=\frac{w_{1}\left(1-w_{1}\right)}{T},
\end{gathered}
$$

$$
1-G_{n \sigma}^{2} \frac{\partial \Sigma_{n \sigma}}{\partial G_{n \sigma}}=\frac{\left(1+G_{n \sigma} \Sigma_{n \sigma}\right)\left(1+G_{n \sigma}\left[\Sigma_{n \sigma}-U\right]\right)}{1+G_{n \sigma}\left(2 \Sigma_{n \sigma}-U\right)} .
$$

Substituting these derivatives into Eq. (36) and performing some straightforward simplifications that involve the quadratic equation that the self energy satisfies 6 finally yields

$$
\left[T \frac{\partial}{\partial h}+\langle w\rangle\right] G_{q \sigma}\left(i \omega_{n}\right)=\frac{\Sigma_{n \sigma}}{U} G_{q \sigma}\left(i \omega_{n}\right) .
$$

Now we are ready to perform the analytic continuation. First we substitute Eq. (40) into Eq. (31) and we note that the sum over $j$ cancels the factor of $1 / N$

$$
\begin{aligned}
\bar{L}_{12}^{P}\left(i \nu_{l}\right) & =-\frac{\pi T^{2}}{2} \sum_{n} \sum_{q \sigma} \mathbf{v}_{q \alpha} \mathbf{v}_{q \beta} \\
& \times\left[\Sigma_{\sigma}\left(i \omega_{n}\right)+\Sigma_{\sigma}\left(i \omega_{n+l}\right)\right] G_{q \sigma}\left(i \omega_{n}\right) G_{q \sigma}\left(i \omega_{n+l}\right) .
\end{aligned}
$$

Next, we rewrite the sum over Matsubara frequencies as a contour integral and perform the analytic continuation in the exact same way as before. If we then evaluate $L_{12}^{P}$ we find

$$
\begin{aligned}
L_{12}^{P} & =\lim _{\nu \rightarrow 0}-\frac{T \sigma_{0}}{2 e^{2} \nu} \int_{-\infty}^{\infty} d \omega \int_{-\infty}^{\infty} d \epsilon \rho(\epsilon) \\
& \times \operatorname{Re}\left[f ( \omega ) \left\{[\Sigma(\omega)+\Sigma(\omega+\nu)] G_{q}(\omega) G_{q}(\omega+\nu)\right.\right. \\
& \left.-\left[\Sigma^{*}(\omega)+\Sigma(\omega+\nu)\right] G_{q}^{*}(\omega) G_{q}(\omega+\nu)\right\} \\
& +f(\omega+\nu)\left\{\left[\Sigma^{*}(\omega)+\Sigma(\omega+\nu)\right] G_{q}^{*}(\omega) G_{q}(\omega+\nu)\right. \\
& \left.\left.-\left[\Sigma^{*}(\omega)+\Sigma^{*}(\omega+\nu)\right] G_{q}^{*}(\omega) G_{q}^{*}(\omega+\nu)\right\}\right] .
\end{aligned}
$$

Now the integral over $\epsilon$ can be performed and the limit $\nu \rightarrow 0$ can be taken. It becomes

$$
\begin{aligned}
L_{12}^{P} & =\frac{T \sigma_{0}}{e^{2}} \int_{-\infty}^{\infty} d \omega\left(-\frac{d f(\omega)}{d \omega}\right)[\operatorname{Re} \Sigma(\omega) \tau(\omega) \\
& +2 \operatorname{Im} \Sigma(\omega) \operatorname{Im}[\{\omega+\mu-\Sigma(\omega)\} G(\omega)]] .
\end{aligned}
$$

Adding together Eqs. (27) and (43) yields the JonsonMahan result of

$$
L_{12}=\frac{T \sigma_{0}}{e^{2}} \int_{-\infty}^{\infty} d \omega\left(-\frac{d f(\omega)}{d \omega}\right) \tau(\omega) \omega .
$$

Our final derivation is for the thermal conductivity coefficient $L_{22}$. Like before, we separate this into pieces corresponding to the kinetic energy and the potential energy: $L_{22}=L_{22}^{K K}+L_{22}^{K P}+L_{22}^{P K}+L_{22}^{P P}$. Due to the symmetry of the terms, we have $L_{22}^{K P}=L_{22}^{P K}$. The kinetic energy piece is simple to calculate. Like in our derivation for $L_{12}^{K}$, the steps are identical to the derivation for $L_{11}$ except we have an extra factor of $(\epsilon-\mu)^{2}$ in Eq. (20). 
Performing the integration over $\epsilon$ and collecting terms finally yields

$$
\begin{aligned}
L_{22}^{K K} & =\frac{T \sigma_{0}}{e^{2}} \int_{-\infty}^{\infty} d \omega\left(-\frac{d f(\omega)}{d \omega}\right) \\
& \times\left\{[\omega-\operatorname{Re} \Sigma(\omega)]^{2} \tau(\omega)+\operatorname{Im} G(\omega) \operatorname{Im} \Sigma(\omega)\right. \\
& -2[\operatorname{Im} \Sigma(\omega)]^{2}+2[\operatorname{Im} \Sigma(\omega)]^{2} \operatorname{Re}\{[\omega+\mu-\Sigma(\omega)] G(\omega)\} \\
& -4[\omega-\operatorname{Re} \Sigma(\omega)] \operatorname{Im} \Sigma(\omega) \operatorname{Im}\{[\omega+\mu-\Sigma(\omega)] G(\omega)\}\} .
\end{aligned}
$$

The derivation for $L_{22}^{K P}=L_{22}^{P K}$ is identical to that of $L_{12}^{P}$ except we have an extra factor of $(\epsilon-\mu)$ in Eq. 42. Performing the integration over $\epsilon$ then produces

$$
\begin{aligned}
L_{22}^{K P} & =\frac{T \sigma_{0}}{e^{2}} \int_{-\infty}^{\infty} d \omega\left(-\frac{d f(\omega)}{d \omega}\right) \\
& \times\{[\omega-\operatorname{Re} \Sigma(\omega)] \operatorname{Re} \Sigma(\omega) \tau(\omega)-\operatorname{Im} G(\omega) \operatorname{Im} \Sigma(\omega) \\
& +2[\operatorname{Im} \Sigma(\omega)]^{2}-2[\operatorname{Im} \Sigma(\omega)]^{2} \operatorname{Re}\{[\omega+\mu-\Sigma(\omega)] G(\omega)\} \\
& +2[\omega-2 \operatorname{Re} \Sigma(\omega)] \operatorname{Im} \Sigma(\omega) \operatorname{Im}\{[\omega+\mu-\Sigma(\omega)] G(\omega)\}\} .
\end{aligned}
$$

The final term we must evaluate is $L_{22}^{P P}$. This is the most complicated term to evaluate and we are unable to do so following the same strategy as employed in the $L_{12}^{P}$ derivation. Instead, we proceed by an alternate method based on the equation of motion (EOM) technique. The EOM for the Fermionic creation and annihilation operators (in the momentum basis) are

$$
\frac{\partial}{\partial \tau} c_{q \sigma}^{\dagger}(\tau)=[\epsilon(q)-\mu] c_{q \sigma}^{\dagger}(\tau)+U \sum_{k} W(k) c_{q+k \sigma}^{\dagger}(\tau),
$$

and

$$
\frac{\partial}{\partial \tau} c_{q \sigma}(\tau)=-[\epsilon(q)-\mu] c_{q \sigma}(\tau)-U \sum_{k} W(k) c_{q-k \sigma}(\tau) .
$$

These EOMs can be employed to express the correlation function of the heat-current operators in terms of derivatives with respect to imaginary time as shown below

$$
\begin{aligned}
& \bar{L}_{22}^{P P}\left(i \nu_{l}\right)=\frac{\pi T U^{2}}{4} \int_{0}^{\beta} d \tau e^{i \nu_{l} \tau} \sum_{q q^{\prime} q^{\prime \prime} q^{\prime \prime \prime} \sigma \sigma^{\prime}} \\
& \left(\mathbf{v}_{q \alpha}+\mathbf{v}_{q^{\prime \prime \prime} \alpha}\right)\left(\mathbf{v}_{q^{\prime} \beta}+\mathbf{v}_{q^{\prime \prime} \beta}\right) \\
\times & \left\langle T_{\tau} W\left(q-q^{\prime \prime \prime}\right) W\left(q^{\prime}-q^{\prime \prime}\right) c_{q \sigma}^{\dagger}(\tau) c_{q^{\prime \prime \prime} \sigma}(\tau)\right. \\
& \left.c_{q^{\prime} \sigma^{\prime}}^{\dagger}(0) c_{q^{\prime \prime} \sigma^{\prime}}(0)\right\rangle \\
= & \pi T \int_{0}^{\beta} d \tau e^{i \nu_{l} \tau} \sum_{q q^{\prime} \sigma \sigma^{\prime}} \mathbf{v}_{q \alpha} \mathbf{v}_{q^{\prime} \beta} \lim _{\tau^{\prime} \rightarrow \tau^{-} \tau^{\prime \prime \prime} \rightarrow \tau^{\prime \prime}-\rightarrow 0^{+}} \lim \\
& \left\langle T_{\tau}\left[\left\{\frac{1}{2}\left(\partial_{\tau}-\partial_{\tau^{\prime}}\right)-\left(\epsilon_{q^{\prime}}-\mu\right)\right\} c_{q^{\prime} \sigma}^{\dagger}(\tau) c_{q^{\prime} \sigma}\left(\tau^{\prime}\right)\right]\right. \\
\times & {\left.\left[\left\{\frac{1}{2}\left(\partial_{\tau^{\prime \prime}}-\partial_{\tau^{\prime \prime \prime}}\right)-\left(\epsilon_{q}-\mu\right)\right\} c_{q \sigma^{\prime}}^{\dagger}\left(\tau^{\prime \prime}\right) c_{q \sigma^{\prime}}\left(\tau^{\prime \prime \prime}\right)\right]\right\rangle . }
\end{aligned}
$$

Now each of the operator averages can be expressed in terms of Green's functions, since the velocity factors guarantee there will be no vertex corrections. Noting further, that the integrals will only contribute if $\alpha=\beta$ finally yields

$$
\begin{aligned}
& \bar{L}_{22}^{P}\left(i \nu_{l}\right)=\pi T \int_{0}^{\beta} d \tau e^{i \nu_{l} \tau} \sum_{q \sigma} v_{q \alpha}^{2} \\
&(45)[ {\left[\frac{1}{2} \partial_{\tau} G_{q \sigma}(\tau) \partial_{\tau} G_{q \sigma}(-\tau)-\frac{1}{4} \partial_{\tau}^{2} G_{q \sigma}(\tau) G_{q \sigma}(-\tau)\right.} \\
&-\frac{1}{4} G_{q \sigma}(\tau) \partial_{\tau}^{2} G_{q \sigma}(-\tau) \\
&+\left(\epsilon_{q}-\mu\right)\left\{G_{q \sigma}(\tau) \partial_{\tau} G_{q \sigma}(-\tau)-\partial_{\tau} G_{q \sigma}(\tau) G_{q \sigma}(-\tau)\right\} \\
&\left.-\left(\epsilon_{q}-\mu\right)^{2} G_{q \sigma}(\tau) G_{q \sigma}(-\tau)\right] .
\end{aligned}
$$

We need to be able to produce expressions for the derivatives of the Green's functions. We do so by first writing the Green's function as a Fourier series over the Matsubara frequencies, and then taking the derivative into the (Matsubara summation. This may appear to be math(ematically unsound, but we do so by adding and subtracting the known $\delta(\tau)$ behavior of the derivative, to regularize the summation. Since we are interested only in $0<\tau<\beta$, this procedure has no convergence issues. Likewise, one is also able to take the second derivative in this fashion. We find for $0<\tau<\beta$

$$
\begin{aligned}
\partial_{\tau} G_{q \sigma}(\tau) & =-\left(\epsilon_{q}-\mu\right) G_{q \sigma}(\tau) \\
& -T \sum_{m} e^{-i \omega_{m} \tau} \frac{\Sigma_{m \sigma}}{i \omega_{m}+\mu-\Sigma_{m \sigma}-\epsilon_{q}}, \\
\partial_{\tau}^{2} G_{q \sigma}(\tau) & =+\left(\epsilon_{q}-\mu\right)^{2} G_{q \sigma}(\tau) \\
& +\left(\epsilon_{q}-\mu\right) T \sum_{m} e^{-i \omega_{m} \tau} \frac{\Sigma_{m \sigma}}{i \omega_{m}+\mu-\Sigma_{m \sigma}-\epsilon_{q}} \\
& +T \sum_{m} e^{-i \omega_{m} \tau} \frac{i \omega_{m} \Sigma_{m \sigma}}{i \omega_{m}+\mu-\Sigma_{m \sigma}-\epsilon_{q}},
\end{aligned}
$$

with similar formulae for $G_{q \sigma}(-\tau)$. Substituting the derivatives from Eq. (51) into Eq. (50), and then simplifying the result finally produces

$$
\begin{aligned}
& \bar{L}_{22}^{P P}\left(i \nu_{l}\right)=-\frac{\pi T^{2}}{4} \sum_{n} \sum_{q \sigma} v_{q \alpha}^{2} \\
& \times\left[\left\{\Sigma_{\sigma}\left(i \omega_{n}\right)+\Sigma_{\sigma}\left(i \omega_{n+l}\right)\right\}^{2} G_{q \sigma}\left(i \omega_{n}\right) G_{q \sigma}\left(i \omega_{n+l}\right)\right. \\
& \left.+\Sigma_{\sigma}\left(i \omega_{n+l}\right) G_{q \sigma}\left(i \omega_{n}\right)+\Sigma_{\sigma}\left(i \omega_{n}\right) G_{q \sigma}\left(i \omega_{n+l}\right)\right] .
\end{aligned}
$$

It is easy to understand the first terms in this expression, as they are what one would naively recover when following the same Wick analysis that was done previously for $L_{12}^{P}$. We have not been able to discover a direct operator-based derivation of the second terms, but they are critical for providing the right answer for $L_{22}^{P P}$. Performing the analytic continuation and simplifying yields our final result 


$$
\begin{aligned}
L_{22}^{P P} & =\frac{T \sigma_{0}}{e^{2}} \int_{-\infty}^{\infty} d \omega\left(-\frac{d f(\omega)}{d \omega}\right) \\
& \times\left\{[\operatorname{Re} \Sigma(\omega)]^{2} \tau(\omega)-2[\operatorname{Im} \Sigma(\omega)]^{2}+\operatorname{Im} \Sigma(\omega) \operatorname{Im} G(\omega)\right. \\
& +2[\operatorname{Im} \Sigma(\omega)]^{2} \operatorname{Re}\{[\omega+\mu-\Sigma(\omega)] G(\omega)\} \\
& +4 \operatorname{Re} \Sigma(\omega) \operatorname{Im} \Sigma(\omega) \operatorname{Im}\{[\omega+\mu-\Sigma(\omega)] G(\omega)\}\}
\end{aligned}
$$

Summing together Eq. (45), twice Eq. (46), and Eq. (53) gives the Mott form

$$
L_{22}=\frac{T \sigma_{0}}{e^{2}} \int_{-\infty}^{\infty} d \omega\left(-\frac{d f(\omega)}{d \omega}\right) \tau(\omega) \omega^{2} .
$$

We can also generalize the original Jonson-Mahan argument to prove relations between $L_{21}$ and $L_{11}$ and between $L_{22}$ and $L_{12}$. Our method is different from their proof and relies on the infinite-dimensional limit, but one could proceed in their fashion if desired. We begin with the generalized two-particle correlation function

$$
\begin{aligned}
& F_{\alpha \beta}\left(\tau, \tau^{\prime}, \tau^{\prime \prime}, \tau^{\prime \prime \prime}\right)= \\
& \sum_{q q^{\prime} \sigma \sigma^{\prime}} \mathbf{v}_{q \alpha} \mathbf{v}_{q^{\prime} \beta}\left\langle T_{\tau} c_{q \sigma}^{\dagger}(\tau) c_{q \sigma}\left(\tau^{\prime}\right) c_{q^{\prime} \sigma^{\prime}}^{\dagger}\left(\tau^{\prime \prime}\right) c_{q^{\prime} \sigma^{\prime}}\left(\tau^{\prime \prime \prime}\right)\right\rangle .
\end{aligned}
$$

In the infinite-dimensional limit, the two-particle correlation function is expressed by just its bare bubble because the irreducible charge vertex has a different symmetry than $\mathbf{v}_{q}$. Hence, we immediately learn that

$$
\begin{aligned}
& F_{\alpha \beta}\left(\tau, \tau^{\prime}, \tau^{\prime \prime}, \tau^{\prime \prime \prime}\right) \\
= & -\sum_{q \sigma} \mathbf{v}_{q \alpha}^{2} \delta_{\alpha \beta} G_{q \sigma}\left(\tau^{\prime \prime \prime}-\tau\right) G_{q \sigma}\left(\tau^{\prime}-\tau^{\prime \prime}\right) .
\end{aligned}
$$

But

$$
G_{q \sigma}(\tau)=\int d \omega A(k, \omega) e^{-\omega \tau}[1-f(\omega)],
$$

for $\tau>0$ and

$$
G_{q \sigma}(\tau)=\int d \omega A(k, \omega) e^{-\omega \tau}[-f(\omega)],
$$

for $\tau<0$. Substituting into Eq. (56), then yields

$$
\begin{aligned}
& F_{\alpha \beta}\left(\tau, \tau^{\prime}, \tau^{\prime \prime}, \tau^{\prime \prime \prime}\right)=\frac{\delta_{\alpha \beta}}{2 d} \int d \epsilon \rho(\epsilon) \int d \omega \int d \omega^{\prime} \\
\times & A(\epsilon, \omega) A\left(\epsilon, \omega^{\prime}\right) e^{\omega\left(\tau-\tau^{\prime \prime \prime}\right)-\omega^{\prime}\left(\tau^{\prime}-\tau^{\prime \prime}\right)} f(\omega)\left[1-f\left(\omega^{\prime}\right)\right] .
\end{aligned}
$$

Using this function we can construct the relevant "polarization operators". Recalling the EOM in Eqs. (47) and (48) shows that

$$
\lim _{\tau^{\prime} \rightarrow \tau^{-}} \frac{1}{2}\left(\frac{\partial}{\partial \tau}-\frac{\partial}{\partial \tau^{\prime}}\right) \sum_{q \sigma} \mathbf{v}_{q} c_{q \sigma}^{\dagger}(\tau) c_{q \sigma}\left(\tau^{\prime}\right)=\mathbf{j}_{Q}(\tau) .
$$

The Jonson-Mahan theorem will hold for any Hamiltonian that satisfies Eq. (60) (which follows from the relevant commutators and equations of motion). This identity holds true for the Falicov-Kimball model, the static
Holstein model, and the periodic Anderson modella, but it does not hold for either the Hubbard model or the quantum Holstein model, a fact which does not appear to be widely known. The "polarization operators" then become

$$
\bar{L}_{11}=\pi T \int_{0}^{\beta} e^{i \nu_{l} \tau} F\left(\tau, \tau^{-}, 0,0\right),
$$

for the conductivity,

$$
\bar{L}_{12}=\pi T \int_{0}^{\beta} e^{i \nu_{l} \tau} \frac{1}{2}\left(\frac{\partial}{\partial \tau}-\frac{\partial}{\partial \tau^{\prime}}\right) F\left(\tau, \tau^{\prime}, 0,0\right),
$$

(in the limit where $\tau^{\prime} \rightarrow \tau^{-}$) for the thermopower, and

$$
\begin{aligned}
\bar{L}_{22} & =\pi T \int_{0}^{\beta} e^{i \nu_{l} \tau} \frac{1}{4}\left(\frac{\partial}{\partial \tau}-\frac{\partial}{\partial \tau^{\prime}}\right)\left(\frac{\partial}{\partial \tau^{\prime \prime}}-\frac{\partial}{\partial \tau^{\prime \prime \prime}}\right) \\
& \times F\left(\tau, \tau^{\prime}, \tau^{\prime \prime}, \tau^{\prime \prime \prime}\right),
\end{aligned}
$$

(in the limit where $\tau^{\prime} \rightarrow \tau^{-}, \tau^{\prime \prime \prime} \rightarrow \tau^{\prime \prime}$, and $\tau^{\prime \prime} \rightarrow 0^{+}$) for the thermal conductivity. Because of Eq. (59), the analytic continuation is trivial (one first converts from imaginary time to Matsubara frequencies and then performs the Wick rotation to the real frequency axis), and if we note the identity

$$
f(\omega)-f(\omega+\nu)=-f(\omega)[1-f(\omega+\nu)]\left[e^{-\beta \nu}-1\right],
$$

then we can easily compute that

$$
L_{11}=\frac{T \sigma_{0}}{e^{2}} \int d \epsilon \rho(\epsilon) \int d \omega\left(-\frac{d f(\omega)}{d \omega}\right) A^{2}(\epsilon, \omega),
$$

for the conductivity,

$$
L_{12}=\frac{T \sigma_{0}}{e^{2}} \int d \epsilon \rho(\epsilon) \int d \omega\left(-\frac{d f(\omega)}{d \omega}\right) A^{2}(\epsilon, \omega) \omega,
$$

for the thermopower, and

$$
L_{22}=\frac{T \sigma_{0}}{e^{2}} \int d \epsilon \rho(\epsilon) \int d \omega\left(-\frac{d f(\omega)}{d \omega}\right) A^{2}(\epsilon, \omega) \omega^{2},
$$

for the thermal conductivity. This proves Mott's form for the thermal transport [since $\int d \epsilon \rho(\epsilon) A^{2}(\epsilon, \omega)=\tau(\omega)$ ].

\section{NUMERICAL RESULTS}

We present results first for a case where the filling of the localized electrons is a constant. We choose the symmetric case of $\langle w\rangle=1 / 2$ and half filling $\rho_{e}=1$ for the electrons. We perform calculations for two cases: (i) $U=1$ which is a moderately correlated metal and (ii) $U=2$ which is a strongly correlated insulator. 


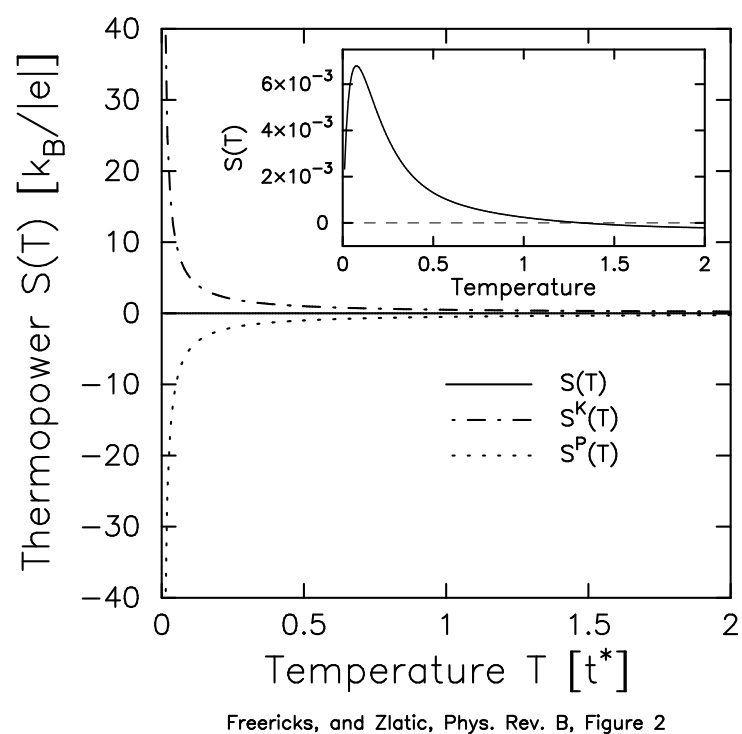

FIG. 2. Thermopower for the case $U=1, w_{1}=0.5$, and $\rho_{d}=1$. The main panel shows the two different contributions from the kinetic and potential energy pieces of the heat current and the inset shows the net thermopower. Note how the two pieces are large and nearly cancel to produce $S$, and how there is a sign change near $T \approx 1.4$.

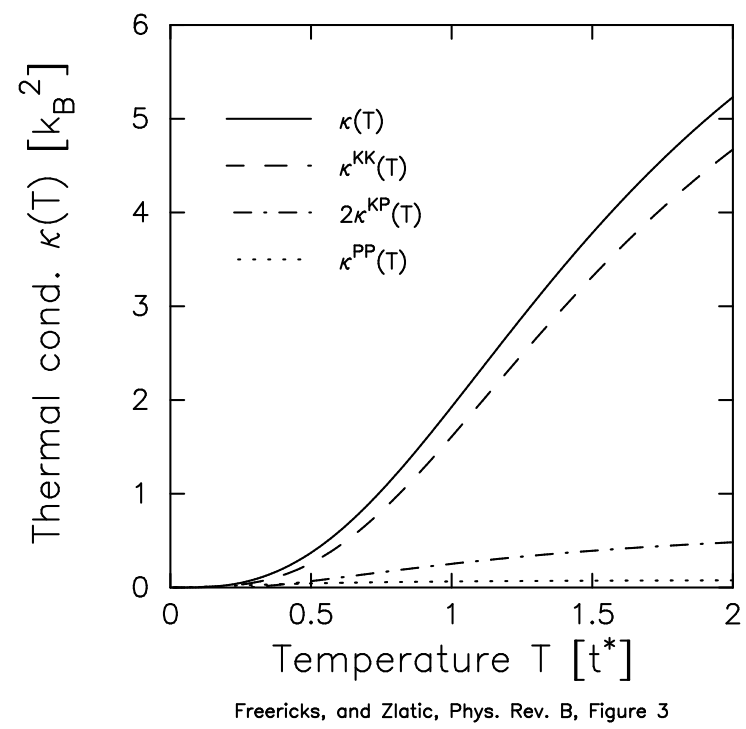

FIG. 3. Thermal conductivity for the case $U=1, w_{1}=0.5$, and $\rho_{d}=1$. The plot shows the different contributions from the kinetic and potential energy pieces of the heat current. Note how the thermal conductivity is essentially described by the kinetic-energy-only piece for moderate correlation strength.

The thermopower behaves as expected - it vanishes for large and small temperature, it has an electron-like peak at lower temperatures and a sign change at a temperature on the order of half the bandwidth. What is surprising is that there is such a large compensation between the kinetic and potential energy pieces of the thermopower to produce the net thermopower (note the three order of magnitude difference in the scales for the main figure and the inset). The thermal conductivity also appears as expected. We can see that while the contributions from the potential energy are critical in determining the right thermopower, they have a relatively mild effect in the thermal conductivity for a moderately correlated metal (note how close the total thermal conductivity is to the kinetic-energy-only contribution).

As we increase the correlation strength, so that the interacting density of states has a gap and the system is a correlated insulator, the behavior of the thermal transport changes.

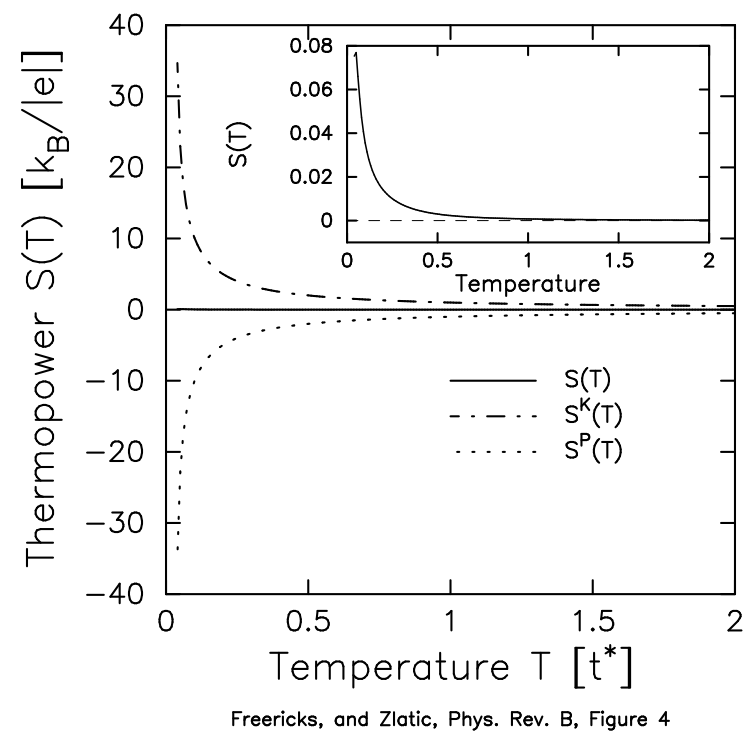

FIG. 4. Thermopower for the case $U=2, w_{1}=0.5$, and $\rho_{d}=1$. The main panel shows the two different contributions from the kinetic and potential energy pieces of the heat current and the inset shows the net thermopower. Note how the two pieces are large and nearly cancel to produce $S$ and how the thermopower appears to diverge as $T$ becomes small (calculations run into numerical problems as $T \rightarrow 0$ ). 


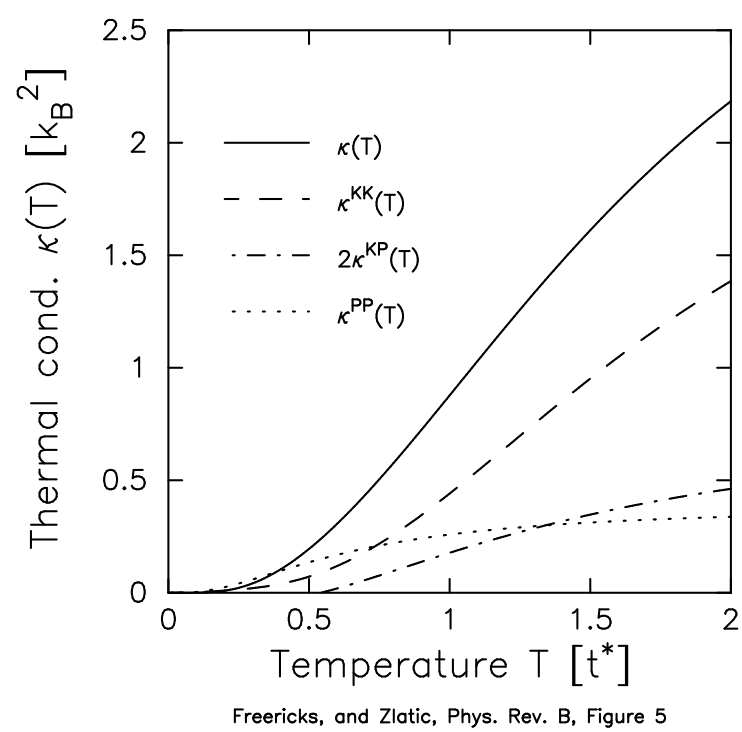

FIG. 5. Thermal conductivity for the case $U=2, w_{1}=0.5$, and $\rho_{d}=1$. The plot shows the different contributions from the kinetic and potential energy pieces of the heat current. Note how the potential energy terms become increasingly important.

The thermopower has the characteristic insulating behavior here, with what appears to be a divergence as $T \rightarrow 0$. The divergence arises from the presence of a gap in the single-particle spectrum-both $L_{11}$ and $L_{12}$ approach zero exponentially in $T$ (with the same exponent), but the ratio is proportional to the size of the gap, so $S \rightarrow C / T+B$ as $T \rightarrow 0$ (similar results have been found when applying scaling theory to the Anderson transition 13 ). Here this is "allowed" thermodynamically, because the "ground-state" of the insulating phase has nonzero entropy, since we forced the system into the paramagnetic insulating phase. In a real system, however, there must be a transition to a ground state where the entropy is quenched. In such a case, one would expect to see a large peak in the thermopower at low energies, with the thermopower ultimately going to zero as $T \rightarrow 0$. We know of no real correlated insulator that has a diverging thermopower as $T \rightarrow 0$.

The computation at very small values of $T$ is difficult because the electrical conductivity (or equivalently $L_{11}$ ) approaches zero and there are numerical difficulties associated with properly calculating the conductivity in this regime due to the cancellation of two large and nearly equal numbers. Since the thermopower requires the electrical conductivity, if that cannot be calculated accurately then spurious behavior will be seen in the thermopower. The thermal conductivity looks similar to the weaker correlated case, except it appears to go to zero at a nonzero temperature which is the expected behavior for a correlated insulator with a gap and the potential energy pieces become increasingly more important (particularly at low temperature).

In both of these half-filled cases, that of a correlated metal and a correlated insulator, the low-temperature thermopower is determined by a slightly larger contribution from the kinetic energy piece of the heat current than from the potential energy piece of the heat current. The thermal conductivity, on the other hand has an evolution of going from a result nearly completely determined by the kinetic energy only piece of the heat current correlation functions to one where the potential energy pieces of the heat current contribute progressively more and more to the total thermal conductivity.

Next we present results for the case where the total filling $\rho_{e}+\langle w\rangle=1.5$ is a constant but the electrons can change from localized to itinerant (i.e., we fix the total electron concentration not the individual electron concentrations). We choose values of the parameters 14 where the system has a sharp transition from a state at high temperature that has large f-occupancy $(\langle w\rangle \approx 0.36$ for $0.2<T<0.8)$, to a state at low temperature with no f-electrons (the crossover occurs near $T=0.04$ ). We find that the results do not depend too strongly on the parameters in this regime, and choose $E_{f}=-0.7$ and $U=4$ as a canonical system that is similar to $\mathrm{YbInCu}_{4}$. The main difference from the symmetric case studied above is that the localized electron filling goes to zero as $T \rightarrow 0$. Hence both the kinetic and potential contributions to the thermopower become small in this limit, and there is no large cancellation between two nearly equal numbers to determine the thermopower. We see that in the thermal conductivity, the contributions from the mixed kinetic and potential energy pieces are the most important, which is an indication of the strengthening of the correlations in the system. This feature is hard to see from the shape of the thermal conductivity itself.

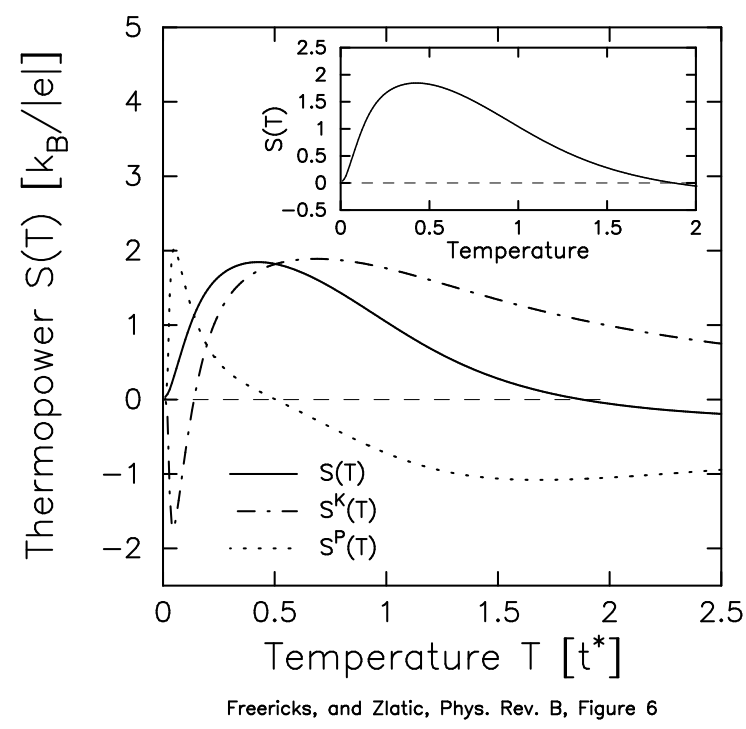


FIG. 6. Thermopower for the case $U=4, w_{1}+\rho_{d}=1.5$, and $E_{F}=-0.7$. The main panel shows the two different contributions from the kinetic and potential energy pieces of the heat current and the inset shows the net thermopower. Note the absolute scale for the thermopower is much larger here.

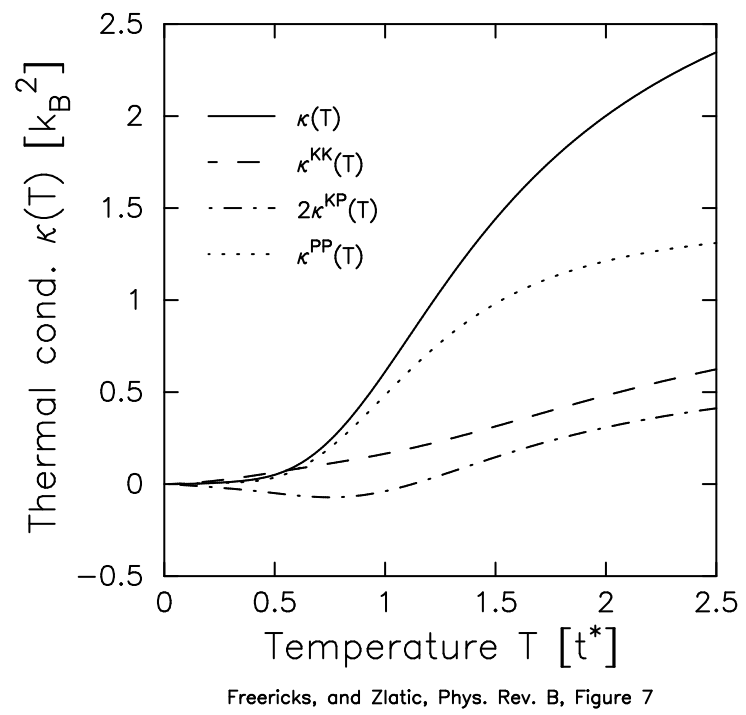

FIG. 7. Thermal conductivity for the case $U=4$, $w_{1}+\rho_{d}=1.5$, and $E_{F}=-0.7$. The plot shows the different contributions from the kinetic and potential energy pieces of the heat current.

The thermopower has an interesting exchange of importance of the different pieces of the heat current as temperature is varied. For high temperatures, the thermopower is determined by both parts of the heat current and a compensation effect is important. In the moderate temperature regime, the thermopower is dominated by kinetic-energy pieces, which then give way to the potential energy domination at low temperature, that eventually shrinks as $T \rightarrow 0$ and the thermopower vanishes.

Note, would need to reverse the sign of the thermopower to describe $\mathrm{YbInCu}_{4}$, since its charge carriers are holes rather than electrons. We should also remark that the thermopower of the Falicov-Kimball model does not have a low-energy peak associated with the "valence-change" transition-such a sharp peak occurs in $\mathrm{YbInCu}_{4}$-like systems because of hybridization effects not included in this model. Consequently, the thermopower also does not have a low-temperature sign change as seen in the experimental data 15 (a sign change occurs at $T \approx 2$ ), but we see that if one could reduce the potential energy piece of the thermal current, then the kinetic energy contributions to the thermopower could cause a sign change to occur. This cannot happen in a pure Falicov-Kimball model though, because of the JonsonMahan theorem. The sign change for the pure FalicovKimball model generically occurs at much larger values of temperature, on the order of the bandwidth. As re- gards the thermal conductivity, we find $\kappa$ is dominated by the potential-energy piece as $T \rightarrow 0$ and the mixed piece yields a negative contribution over a wide temperature range.

\section{CONCLUSIONS}

We have examined thermal transport in the spin-onehalf Falicov-Kimball model. We chose this model because the transport properties can be solved exactly, and they provide an alternate proof of the Jonson-Mahan theorem for the thermopower. We provide the proof in two different ways. The first is a brute-force application of the dynamical mean field theory to calculate all relevant correlation functions and combine all terms to yield the final expressions for the thermal transport coefficients. The second is based largely on the techniques of Jonson and Mahan, but one can determine the important "generalized polarization" functions exactly in the large dimensional limit. Here we extend the Jonson-Mahan arguments to show analogous results hold for the thermal conductivity as well.

Our formulation also allows us to decompose the contributions to the thermopower and the thermal conductivity into the respective contributions from the kinetic energy piece and the potential energy piece of the thermal current. We find that generically, these pieces are large and opposite in sign for the thermopower so that thermal transport carried by the kinetic heat current is almost completely compensated by the potential heat current producing a small net thermopower. For the thermal conductivity, we see an evolution of the transport being dominated first by kinetic energy terms and then potential energy terms as the strength of the correlations increase. We note, that because the kinetic-energy contribution to the thermopower can be straightforwardly determined for a number of models, any Hamiltonian that satisfies the Jonson-Mahan theorem can be separated into its kinetic and potential pieces for the thermopower by simply subtracting the kinetic energy piece from the Jonson-Mahan result.

\section{ACKNOWLEDGMENTS}

We would like to acknowledge stimulating discussions with G. Czycholl, B. Letfulov, G. Mahan, R. Römer and C. Villagonzalo. This work was supported by the National Science Foundation under grant DMR-9973225.

${ }^{1}$ M. Jonson and G.D. Mahan, Phys. Rev. B 21, 4223 (1980); ibid. 42, 9350 (1990). 
${ }^{2}$ G.D. Mahan, Phys. Rev. B 56, 11833, (1997); Solid State Phys. 51, 81 (1998).

${ }^{3}$ G.V. Chester and A. Thellung, Proc. Phys. Soc. (London) 771005 (1961); N.F. Mott and E.A. Davis, Electronic Processes in Non-Crystalline Materials, (Clarendon, Oxford, 1971) p. 47.

${ }^{4}$ L.M. Falicov and J.C. Kimball, Phys. Rev. Lett. 22, 997 (1969); R. Ramirez, L.M. Falicov and J.C. Kimball, Phys. Rev. B 2, 3383 (1970).

${ }^{5}$ W. Metzner and D. Vollhardt, Phys. Rev. Lett. 62, 324 (1989).

${ }^{6}$ U. Brandt and C. Mielsch, Z. Phys. B 75, 365 (1989); 79, 295 (1990); U. Brandt, A. Fledderjohann, and G. Hülsenbeck, ibid. 81, 409 (1990); U. Brandt and C. Mielsch, ibid. 82, 37 (1991).

${ }^{7}$ J.K. Freericks and V. Zlatić, Phys. Rev. B 58, 322 (1998); V. Zlatić, J.K. Freericks, R. Lemański, and G. Czycholl, Phil. Mag. B to appear (2001).

${ }^{8}$ R. Kubo, J. Phys. Soc. Japan 12, 570 (1957); D.A. Greenwood, Proc. Phys. Soc. (London) 71, 585 (1958).

${ }^{9}$ G.D. Mahan, Many-Particle Physics, (New York, Plenum, 1990).

${ }^{10}$ A. Khurana, Phys. Rev. Lett., 64, 1990 (1990).

${ }^{11}$ E. Müller-Hartmann, Z. Phys. B 74, 507 (1989); ibid. 76 211 (1989).

12 Th. Pruschke, D.L. Cox, and M. Jarrell, Phys. Rev. B 47, 3553 (1993); Th. Pruschke, D.L. Cox, and M. Jarrell, Europhys. Lett. 21, 593 (1993).

13 C. Villagonzalo and R. Römer, Ann. Phys. (Leipzig) 7, 394 (1998); C. Villagonzalo, R. Römer, and M. Schreiber, Eur. Phys. J. (France) B 12, 179 (1999); Ann. Phys. (Leipzig) 8, Spec. Issue SI-269 (1999); C. Villagonzalo, R. Römer, M. Schreiber, and A. MacKinnon, Phys. Rev. B 62, 1646 (2000).

${ }^{14}$ V. Zlatić and J.K. Freericks, in Open Problems in Strongly Correlated Electron Systems, edited by J. Bonča, P. Prelovšek, A. Ramšak, and S. Sarkar (Dordrecht, Kluwer, 2001) p. 371; V. Zlatić and J.K. Freericks, submitted to Acta Physica Polonica B.

${ }^{15}$ M. Očko, D. Drobac, J.L. Sarrao, and Z. Fisk, Phys. Rev. B 64, 085103 (2001); M. Očko and J.L. Sarrao, Physica B (to appear). 